\title{
Stimulus control and function of arm and wall travel by rats on a radial arm floor maze
}

\author{
WILLIAM TIMBERLAKE, JOSEPH LEFFEL, and CYNTHIA M. HOFFMAN \\ Indiana University, Bloomington, Indiana
}

\begin{abstract}
Hoffman, Timberlake, Leffel, and Gont (1999) concluded that the tactic of effective trail following (in the form of arm and wall travel), rather than distance minimizing, central-place search, or random search, best characterized the locomotion of rats on a radial arm maze placed flat on the floor of an arena (a floor RAM). The present experiments analyzed further the stimulus control and function of arm and wall travel. Experiment 1 showed that arm travel was controlled more by the edge of a maze arm than by its surface. Experiment 2 showed that rats with whiskers clipped on one side traveled along arms less and along walls more than did intact rats. Experiment 3 showed that maze arms increased search effectiveness and decreased suppression of locomotion by bright light and a novel environment. The results support the hypothesis that arm and wall travel are based on mechanisms of trail following, which, in natural settings, contribute to food finding and regulation of social relations and fear.
\end{abstract}

Hoffman, Timberlake, Leffel, and Gont (1999) examined the potential contributions of four locomotor tactics to effective food gathering by rats on a radial arm maze (RAM). The tactics were distance minimizing, centralplace search, trail following, and random (meandering) search. These tactics, in conjunction with a win-shift rule, make identical predictions about locomotor behavior on an elevated RAM, so Hoffman et al. placed the arms and center platform of a six-arm RAM flat on the floor of a large arena. Because the rats were free to approach the food cups from any direction, on or off the maze, the predictions of these locomotor tactics could be contrasted.

In a standard-arm RAM on the floor, rats could distance minimize by traveling from the central platform to a cup and then in a circular route (a series of flat arcs) between cups. They could show central-place search or trail following by moving from the center to each cup and back. The rats also could search randomly, or along walls to food cups (thigmotactic search), or could fail to find food cups in an efficient manner. After exploring freely on and off maze arms during the initial trials, the rats predominantly traveled along maze arms to visit the food cups. Arm travel dominated even when the distance required to search all the cups was three times that of a direct route and even when the food cups were never baited. When no maze arms were present, the rats followed the arena walls between cup visits.

This study was supported in part by National Science Foundation Grants IBN 9408366, IBN-9817175, and BIR 9413220. C.M.H. was supported by National Research Service Award IF32MH10662. The authors thank Tammi Nelson and Jason Hesting for their technical assistance. Correspondence should be addressed to W. Timberlake, Department of Psychology, Indiana University, Bloomington, IN 47405 (e-mail: timberla@indiana.edu).
The pervasiveness of arm travel in the presence of a variety of more efficient routes to food (see also Roche \& Timberlake, 1998) suggests that arm travel is controlled in part by species-typical perceptual-motor mechanisms related to the extensive trail following shown by colonies of wild rats (Calhoun, 1962; Telle, 1966). This trail-following hypothesis raises several empirical and theoretical issues. The first question is, What stimulus characteristics control arm (and wall) travel? Because Hoffman et al. (1999) failed to show a reliable effect of odor in guiding locomotion on a floor RAM, we assumed that visual and tactual cues were primary candidates for controlling stimuli.

The second question concerns the relation between arm and wall travel in rats. Arm travel is usually associated with finding food. Wall travel is usually interpreted as fear-related antipredator behavior that keeps the rat near cover and away from large open areas (Barnett, 1975; Simon, Dupuis, \& Costentin, 1994; Valle, 1970, 1971). However, the results of Hoffman et al. (1999) suggest that wall travel and arm travel are related and, thus, may both be a function of trail-following mechanisms. For example, Hoffman et al. showed that as rats decreased wall travel over trials or across conditions, they typically increased arm travel, whereas the amount of travel in the open changed very little. In a related finding, when arm travel was eliminated by removing the arms, rats markedly increased wall travel between food cups.

The purpose of the present experiments was to analyze further the sensorimotor control and function of arm travel in order to clarify its potential relation to wall travel in the laboratory and to trail following in natural settings. In Experiment 1, we investigated the extent to which the surface and borders of standard maze arms promoted arm travel. In Experiment 2, we tested the role of tactual stimuli in promoting arm and wall travel by clipping the rats' 
whiskers on one side. In Experiment 3, we tested the role of floor-maze arms in facilitating search behavior under conditions of high illumination and novelty.

\section{EXPERIMENT 1}

The data of Hoffman et al. (1999) and Roche and Timberlake (1998) clearly showed the importance of maze arms on the floor of an arena in guiding locomotion. The purpose of Experiment 1 was to clarify the stimulus characteristics of the maze arms that controlled travel. For example, the texture and reflectance of the painted surface of the arm provided a rat's feet, eyes, and whiskers with cues that distinguished it from the floor of the arena. Visual and tactual stimulation was also provided by vertical borders on either side of the maze arms that projected $1.9 \mathrm{~cm}$ above the top of the $1.9-\mathrm{cm}$-thick arm. Finally, although odor had little apparent effect on locomotion in Hoffman et al.'s study, it seemed important to continue to test for, rather than ignore, its potential contribution (Galef \& Buckley, 1996).

Two experimental and two standard-arm control groups of food-deprived rats were placed on a baited six-arm RAM placed flat on the floor of a large arena. The standard-arm groups provided a baseline of typical arm effects and extended the replicability of the results of Hoffman et al. (1999) that showed predominant arm travel and effective cup visits. Two separate standard-arm groups were used to ensure appropriate comparisons for the edge-only and surface-only experimental groups because of slight differences between the edge-only and surfaceonly groups in terms of the size of the arena $(3.2 \times 2.9 \mathrm{~m}$ vs. $3.6 \times 3.2 \mathrm{~m}$ ) and the maximum duration of each trial ( 5 min vs. $10 \mathrm{~min}$ ).

For the edge-only group, the arms of the floor RAM were reduced to a single free-standing border created by gluing together in an inverted " $T$ " two 70-cm-long pieces of 3.8-cm-wide, $0.5-\mathrm{cm}$-thick hard-finish fiberboard (Masonite) painted gray. Although the free-standing border provided nothing for the rats to walk on, they could maintain visual and whisker contact with the vertical edge by walking beside it on the arena floor. To the extent that visual and tactual inputs from the surface of the maze arm are important, the edge-only condition should interfere with arm travel. On the other hand, if a single vertical edge is a sufficient guide for arm travel, then performance of the edge-only maze group should be similar to that on a standard floor RAM.

For the surface-only group, the arms of the floor RAM were reduced in height but not width by constructing them of $0.5-\mathrm{cm}$-thick painted Masonite without any vertical borders at the edges. The rats could easily walk on top of the 13-cm-wide arms, producing different visual and tactual inputs than were provided by the concrete floor of the arena, but the absence of borders and the thin $0.5-\mathrm{cm}$ height of the floor itself produced a minimal vertical edge. To the extent that arm travel is guided by the tactual/visual substrate, performance on this maze should be similar to that of the standard floor RAM group. If, on the other hand, the continuous vertical edges of a standard maze arm are important to arm travel (Hoffman et al., 1999), this group should show less travel.

\section{Method}

\section{Subjects}

The subjects were 36 naive adult (90-120-day-old) female Sprague-Dawley albino rats (Rattus norvegicus) bred at the Indiana University colony. They were housed singly under a 12:12-h light:dark cycle and fed in their home cages once a day after running to maintain them at $85 \%$ of free-feeding weight. Except as noted, the subjects were treated the same as those in Hoffman et al. (1999). Two rats were eliminated from the surface-only group and 1 rat was eliminated from its standard-arm control group because they failed to leave the central platform on at least two of the first three trials.

\section{Apparatus}

The six-arm radial maze in the standard-arm condition (see Hoffman et al., 1999) resembled a typical elevated maze constructed of plywood with hard fiberboard (Masonite) borders extending $1.9 \mathrm{~cm}$ above the surface on both sides of each arm. The entire maze was painted gray and placed flat on the floor of an arena. Each arm measured $13 \mathrm{~cm}$ wide $\times 70 \mathrm{~cm}$ long. A ceramic food cup, $7.5 \mathrm{~cm}$ in diameter and $5 \mathrm{~cm}$ tall, was placed at the end of each radial arm. The rats could easily take food from the cup while standing on the arm or on the floor next to the arm.

The hexagonal center measured $45 \mathrm{~cm}$ from point to opposite point of the hexagon and had a top of clear Plexiglas covered with $1.2-\mathrm{cm}$ strips of black construction paper spaced $1.2 \mathrm{~cm}$ apart. The edge-only arms were constructed of a single border (Masonite; 3.8 $\mathrm{cm}$ tall $\times 70 \mathrm{~cm}$ long) glued to an identical piece of Masonite that served as a base, all painted gray. The arena was $3.2 \times 2.9 \mathrm{~m}$ for the edge-only group and its standard-arm group. The surface-only arms were constructed of Masonite $(13 \mathrm{~cm}$ wide $\times 70 \mathrm{~cm}$ long $\times 0.5 \mathrm{~cm}$ thick) with no borders, also painted gray. The arena for the surfaceonly group and its standard-arm group was $3.6 \times 3.2 \mathrm{~m}$. Both arenas were divided into $45.7-\mathrm{cm}$ squares by masking tape.

\section{Procedure}

Each rat was tested one trial per day between the hours of 2 and 6 p.m. for a total of 12 days. One $45-\mathrm{mg}$ Bio-Serv pellet was placed in each food cup prior to the beginning of a trial. During the trial, the experimenter drew on a data sheet divided into squares proportional to the squares on the arena floor. The travel pattern that the rat followed on and off of the maze; the experimenter also recorded the cup visits and the time elapsed at the end of the trial. A cup visit was scored when the rat approached and inserted its nose in a food cup. A trial ended when the rat had visited each of the six novel cups and 1 additional minute had elapsed, or when it had made a total of eight visits to food cups, or at the end of $5 \mathrm{~min}$ for the surfaceonly group and control, or $10 \mathrm{~min}$ for the edge-only group and control. At the end of the trial, the rat was gently picked up by the experimenter and returned to the carrier cage.

Measures and methods of analysis were the same as in Hoffman et al. (1999) except the routes were traced twice, rather than three times, before averaging for reliability. To assess how effectively the rats located the available food each trial, we counted the number of novel cups visited out of a maximum of six visits and compared this with the performance of ather groups and with chance. The chance level of choosing novel food cups out of six choices (3.99) was calculated as though the rat were always confronted with six choices on a series of six independent trials (Hoffman et al., 1999). This 
a

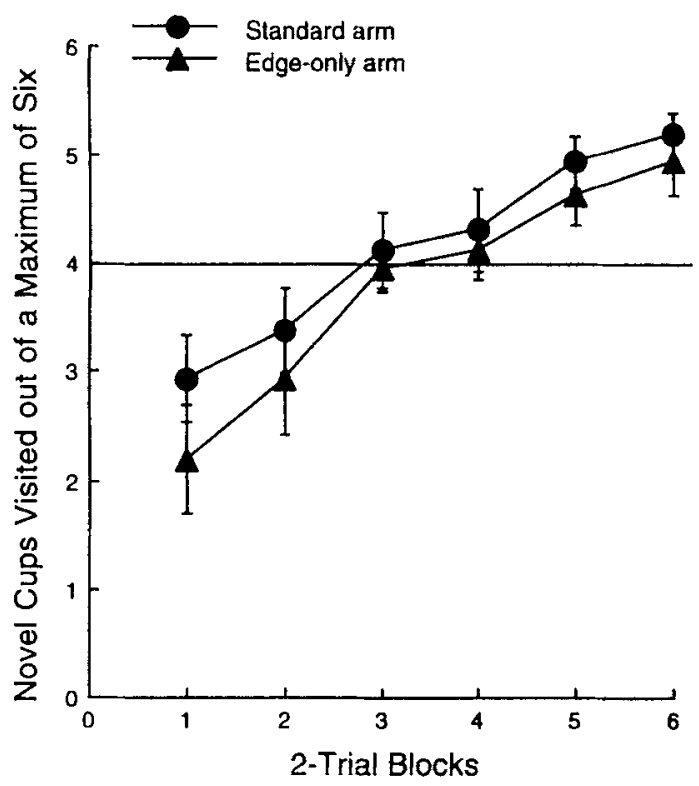

b

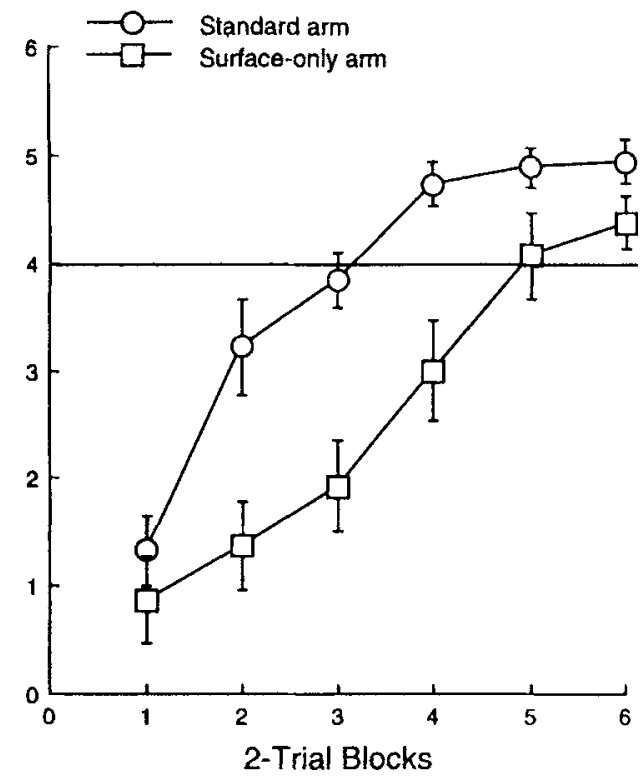

Figure 1. (a) Mean number of novel cups visited in a maximum of six visits on a floor RAM with standard and edgeonly arms, plotted by two-trial blocks. (b) Mean number of novel cups visited in a maximum of six visits on a floor RAM with standard and surface-only arms plotted by two-trial blocks. The solid line in each panel shows the number of novel cup visits expected by chance in six cup visits. Data are from Experiment 1.

was considered a high estimate of chance because it treated the rat as though it always returned to the central platform before its next choice between six equidistant cups. Because the rat did not always return to the center (and so did not have six equidistant choices), the actual likelihood of visiting novel cups by chance is probably less because of a tendency to visit more proximate cups. We also measured the time it took to complete each trial.

We used two measures of the pattern of cup choice: the proportion of successive visits to adjacent cups (dividing the number of successive cup visits by the total number of cup visits on each trial), and the number of arcs (instances in which the rat traveled directly from one cup to the next, without traveling along an arm or the wall or meandering about). A perfect distance minimizer should generate 5 arcs and a proportion of .83 for adjacent cup visit by traveling from the center out to a food cup and then in a circle touching each of the other food cups in succession.

The locomotion of the rats was divided into arm travel (locomotion on or parallel to and within $5 \mathrm{~cm}$ of an arm for a distance of $15 \mathrm{~cm}$ ), wall travel (locomotion parallel to and within $30 \mathrm{~cm}$ of the wall), and travel in the open. We measured arm travel, wall travel, and open travel by tracing our data sheets twice with a pen mouse and averaging the results (see Hoffman et al., 1999). The absolute distance traveled was totaled and the proportion of arm travel, open travel, and wall travel was calculated for each trial for each rat and averaged by trial blocks.

Dependent variables were analyzed using mixed-design analyses of variance (ANOVAs). One-tailed $t$ tests were conducted when comparing the accuracy of rats with the constant chance value of 3.99 and when comparing the travel route of rats with that expected of an optimal forager ( 5 arcs). Except for Experiment 3, data were combined into two-trial blocks for analysis. Transformations were applied as appropriate to reduce the correlation between group means and variances. We used an alpha level of .05 for reporting significant differences. Finally, to determine whether odors left on the maze and floor by the rats that ran early in each subgroup had an effect on the foraging behavior of the rats tested later in each subgroup, we conducted ANOVAs on all dependent variables comparing early rats (the first 2 rats in each subgroup) with late rats (the last 2 rats in each subgroup).

\section{Results}

The results are separated into data concerned primarily with (1) the effectiveness of the rats in finding all the food (number of novel cups visits out of a maximum of six visits, time to complete a trial, and search efficiencynovel cups per meter traveled) and (2) the patterns of locomotion shown in finding food (proportion of successive adjacent cup visits, number of arcs, and proportion of distance traveled along arms and walls and in the open). Because the edge-only and surface-only groups were tested in arenas of slightly different size, we compared each with its own standard-arm control group rather than beginning with an overall analysis.

\section{Cup Visits}

Novel cup visits. Figure la shows that both the edgeonly group and its standard-arm control group increased the number of novel cups visited each trial block during a maximum of six cup visits $[F(5,70)=24.4]$ (there were fewer than six cup visits if the rat exceeded the time limit of the trial). The two groups did not $\operatorname{differ}[F(1,14)<1]$, and there was no interaction. Both groups chose novel 
Table 1

Mean Performance Measures and Standard Errors by Two-Trial Blocks for Experiment 1

\begin{tabular}{|c|c|c|c|c|c|c|c|c|c|c|c|c|c|}
\hline \multirow[b]{2}{*}{ Maze } & \multicolumn{2}{|c|}{ Block 1} & \multicolumn{2}{|c|}{ Block 2} & Block 3 & \multicolumn{2}{|c|}{ Block 4} & \multicolumn{2}{|c|}{ Block 5} & \multicolumn{2}{|c|}{ Block 6} & \multicolumn{2}{|c|}{ Overall } \\
\hline & $M$ & $S E$ & $M$ & $S E$ & $M S E$ & $M$ & $S E$ & $M$ & $S E$ & $M$ & $S E$ & $M$ & $S E$ \\
\hline \multicolumn{14}{|c|}{ (a) Mean Time (in Seconds) to Complete a Trial for the Edge-Only Group and Their Control } \\
\hline Standard arm & 387 & 48 & 267 & 65 & $194 \quad 60$ & 140 & 43 & 127 & 51 & 103 & 28 & 203 & 45 \\
\hline Edge only & 488 & 47 & 357 & 60 & $263 \quad 46$ & 233 & 27 & 122 & 7 & 102 & 13 & 261 & 22 \\
\hline \multicolumn{14}{|c|}{ (b) Mean Time (in Seconds) to Complete a Trial for the Surface-Only Group and Their Control } \\
\hline Standard arm & 300 & 0 & 271 & 13 & $220 \quad 24$ & 180 & 14 & 136 & 15 & 135 & 25 & 207 & 12 \\
\hline Surface only & 300 & 0 & 282 & 12 & $250 \quad 29$ & 227 & 23 & 221 & 22 & 164 & 31 & 241 & 17 \\
\hline \multicolumn{14}{|c|}{ (c) Mean Proportion of Successive Visits to an Adjacent Cup for the Edge-Only Group and Their Control } \\
\hline Standard arm & .32 & .06 & .27 & .06 & .39 .05 & .41 & .05 & .44 & .07 & .50 & .07 & .39 & .04 \\
\hline Edge only & .30 & .09 & .30 & .07 & $.42 \quad .05$ & .47 & .07 & .51 & .06 & .59 & .06 & .43 & .04 \\
\hline \multicolumn{14}{|c|}{ (d) Mean Proportion of Successive Visits to an Adjacent Cup for the Surface-Only Group and Their Control } \\
\hline Standard arm & .12 & .05 & .31 & .06 & $.31 \quad .06$ & .22 & .05 & .33 & .05 & .38 & .05 & .28 & .03 \\
\hline Surface only & .10 & .05 & .21 & .08 & $.22 \quad .08$ & .47 & .08 & .39 & .04 & .41 & .04 & .30 & .03 \\
\hline \multicolumn{14}{|c|}{ (e) Mean Number of Arcs for the Edge-Only Group and Their Control } \\
\hline Standard arm & 0.13 & 0.08 & 0.13 & 0.08 & 0.060 .06 & 0.31 & 0.13 & 0.50 & 0.38 & 0.44 & 0.24 & 0.26 & 0.14 \\
\hline Edge only & 0.0 & 0.0 & 0.19 & 0.09 & 0.380 .16 & 0.56 & 0.36 & 0.38 & 0.18 & 0.38 & 0.13 & 0.31 & 0.10 \\
\hline \multicolumn{14}{|c|}{ (f) Mean Number of Ares for the Surface-Only Group and Their Control } \\
\hline Standard arm & 0.06 & 0.06 & 0.11 & 0.07 & 0.060 .06 & 0.17 & 0.08 & 0.17 & 0.12 & 0.11 & 0.07 & 0.11 & 0.04 \\
\hline Surface only & 0.13 & 0.08 & 0.19 & 0.19 & 0.310 .13 & 0.69 & 0.30 & 0.50 & 0.13 & 1.25 & 0.27 & 0.51 & 0.11 \\
\hline
\end{tabular}

arms significantly above chance at asymptote over the last two blocks of trials [ts $(7)=3.81$ and 5.25].

Figure $1 \mathrm{~b}$ shows that, although both the surface-only group and its standard-arm group also increased the number of novel cups visited each trial block during a maximum of six cup visits $[F(5,75)=55.6]$, the surface-only rats chose significantly fewer total novel cups $[F(1,15)=$ $12.8]$. There also was a significant interaction between groups and blocks of trials $[F(5,75)=3.31]$. The control rats chose novel cups above chance at asymptote over the last two blocks of trials $[t(8)=6.76]$, whereas the surfaceonly rats never chose novel cups significantly above our estimate of chance.

In short, the edge-only group did not differ significantly from its standard-arm control group in choosing novel cups, whereas the surface-only group was less adept in finding food than was its control group. A comparison across trial blocks of the differences between the edge-only group and the surface-only group and their respective standard-arm groups supported the view that the surface-only group found novel cups significantly less well $[F(1,14)=4.99]$.

As discussed in the Method section and in Hoffman et al. (1999), there are two reasons that animals may choose a number of novel cups below our estimate of chance. The first is that they initially may choose fewer than six cups. The second is that our estimate is most appropriate for an elevated maze in which the rats always choose between equally available (equidistant) cups. In the case of a floor maze, choices are frequently made from the area of a food cup, with the result that some cups are considerably closer than others. These unequal distances appear to increase the likelihood of choosing already chosen cups and, thus, decrease the number of novel cups chosen by chance. Because we could not easily estimate this lower level, it is most appropriate to treat our estimated chance level as a performance standard rather than as true chance.

Time. Table la shows that the rats in both the edgeonly group and the rats in its standard-arm control group markedly decreased their log time to complete a trial over trial blocks $[F(5,70)=43.1]$; these groups did not differ significantly, and there was no interaction with trial blocks. Table $\mathrm{lb}$ reveals a similar decrease in the time to complete a trial by the surface-only group and its standardarm control group $[F(5,75)=25.7]$, along with a similar lack of significant difference between these groups and no interaction with trial blocks.

Efficiency. Figure 2 shows that the efficiency of search in terms of novel cups found per meter traversed was similar to that for the novel-cups measure. The rats in the edge-only group and the rats in their standard-arm control group increased their efficiency in finding food over trial blocks $[F(5,70)=27.9]$ and did not differ from each other overall $[F(1,14)=1.54]$. The surface-only group and its standard-arm control group also increased their search efficiency over trial blocks $[F(5,75)=31$.1], but the surface-only group was significantly less efficient than its standard-arm control group overall $[F(1,15)=$ 4.79]. There also was a group $\times$ trial block interaction $[F(5,75)=4.70]$, with the result that the two groups did not differ at the last trial block $[F(1,15)<1]$.

\section{Locomotion Patterns}

It is important to note that the rats in all experimental conditions easily traveled around the arena both on and off 
a

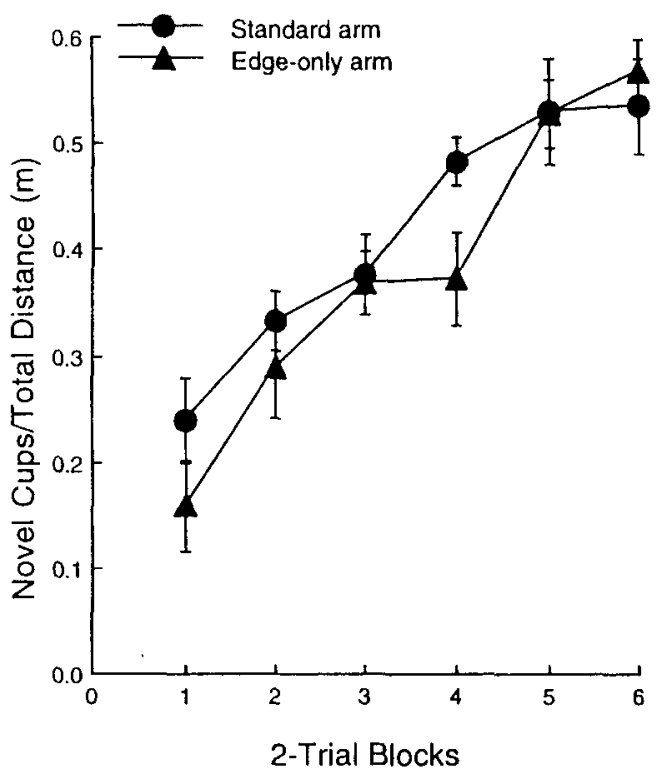

b

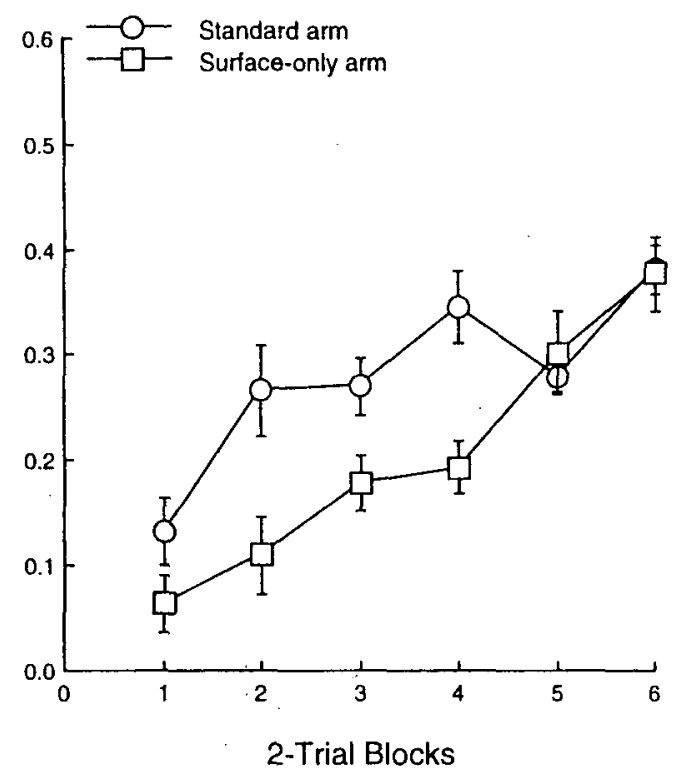

Figure 2. Mean efficiency in visiting novel food cups in terms of cups visited per distance traveled (cups/meter) plotted by two-trial blocks for (a) the rats in the edge-only group and their standard-arm group and (b) the rats in the surface-only group and their standard-arm group. Data are from Experiment 1.

the arms of the floor RAM. Figure 3 shows a modal route for the rats in each of the four groups: the edge-only group and its standard-arm control group, and the surface-only group and its standard-arm control group. Each modal route was constructed by modifying the tracings of one or two sample trials so that they were compatible with the averages of all measures on the last two blocks of trials.

Adjacent cup visits. Table $1 \mathrm{c}$ shows that the edgeonly rats and their standard-arm controls increased their proportion of successive visits to adjacent cups over trial blocks $[F(5,70)=6.52]$, indicating the emergence of a rule-driven search. The two groups did not differ overall, and there was no interaction. Table $1 \mathrm{~d}$ shows that the surface-only group and its standard-arm control group also increased the proportion of successive adjacent visits over trial blocks $[F(5,75)=8.20]$, also with no difference between the groups. However, there was a significant interaction with trial blocks $[F(5,75)=3.07]$ (see the analysis of arcs below).

Number of arcs. To estimate the similarity of the obtained locomotor patterns to that of an ideal distance minimizer, we counted the number of instances per trial in which the rats showed arcs, traveling directly from one cup to the next. Table le shows that the mean number of arcs did not change significantly over trial blocks for the edge-only group and its control group, nor was there a significant group effect or interaction. In contrast, Table if shows a significant increase in arcs over trial blocks for the surface-only rats and their controls $[F(5,75)=5.47]$, combined with a groups effect $[F(1,15)=12.7]$, and a groups $\times$ trial block interaction $[F(5,75)=4.53]$. Followup tests showed that the control group did not change the number of arcs over days, whereas the surface-only group significantly increased arcs $[F(5,35)=5.23]$.

Proportion of distance traveled. Figures $4 \mathrm{a}$ and $4 \mathrm{~b}$ show the $\log$ proportion of distance traveled near maze arms, near walls, and in the open for the edge-only rats and their standard-arm controls. An overall ANOVA showed a significant effect of travel type $[F(2,28)=4.85]$ and trial blocks $[F(5,70)=3.79]$. Summed over both travel type and trial block, there was no difference in log proportions between the edge-only rats and their standardarm controls. Such an overall group difference is unlikely because the proportions of travel types for each rat must sum to one for each rat; however, since the log proportions are less constrained, a difference is possible. Finally, because the three travel types are exhaustive, any comparison that involved significant differences in all three categories either between groups or over trials would need to be viewed descriptively rather than causally. However, there were no such cases.

The edge-only rats traveled approximately equal proportions of their total distance along arms and walls and in the open. However, their standard-arm controls showed differences in travel proportions $[F(2,14)=6.48]$. The proportions of travel along arms and walls were greater than the proportion of travel in the open $[F \mathrm{~s}(1,7)=15.4$ and 6.12 , respectively]. The apparent differences between travel along arms and walls did not reach significance. In comparisons of the types of travel between the edge-only 

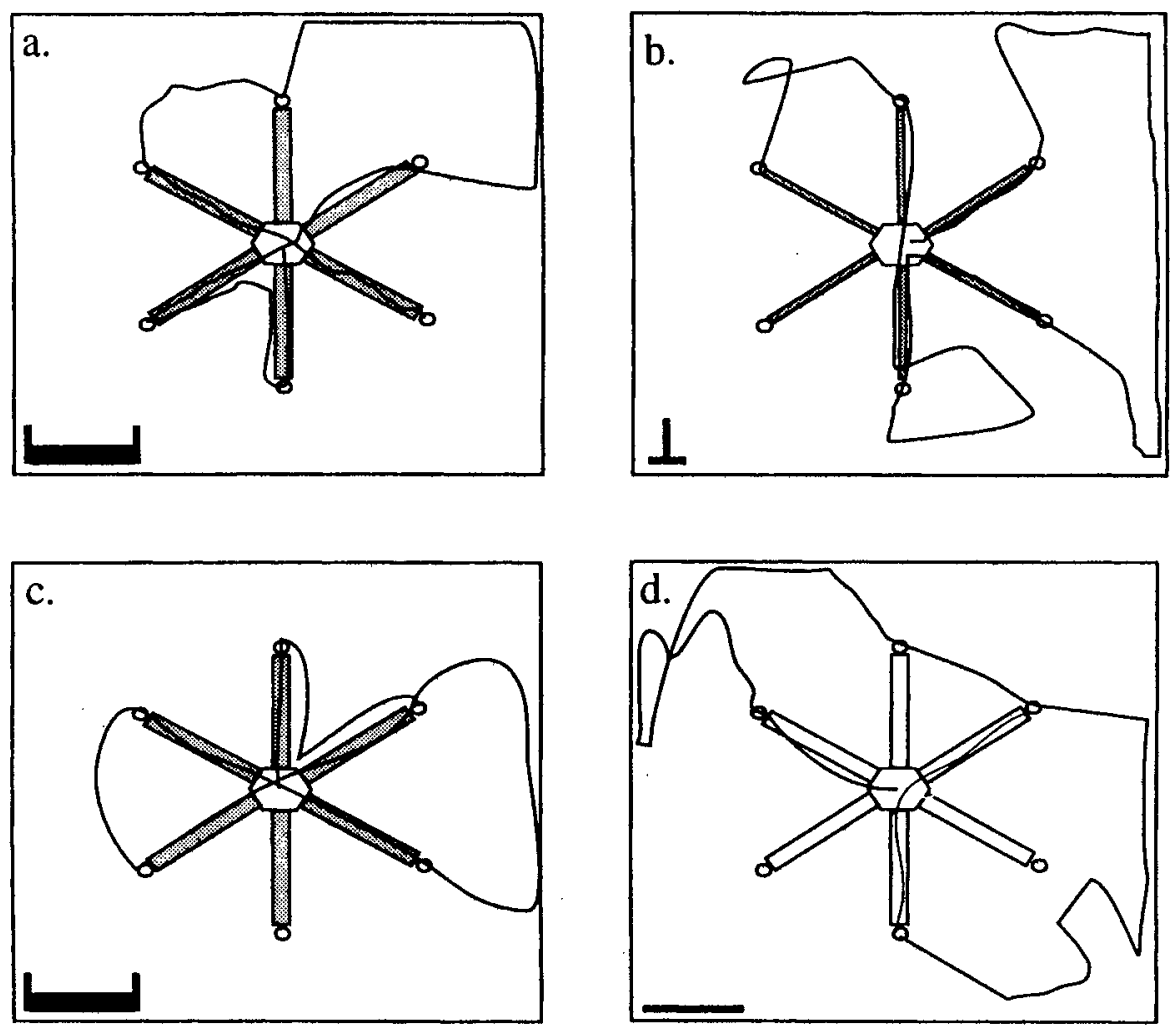

Figure 3. Modal routes on a floor RAM that represent the average measures over the last two blocks of trials for ( $b$ and a) the edge-only rats and their standard-arm group and ( $d$ and $c$ ) the surface-only rats and their standard-arm group. The graphic in the bottom left corner of each panel illustrates the type of arm. Data are from Experiment 1.

group and its control group, the edge-only rats traveled a greater proportion of their distance in the open $[F(1,14)=$ 15.6].

Figures $5 \mathrm{a}$ and $5 \mathrm{~b}$ show a similar but more complex relation between the travel proportions for the surfaceonly group and its standard-arm control group. An overall ANOVA showed a significant effect of travel type $[F(5,75)=4.13]$ and trial blocks $[F(2,30)=7.25]$. Subsequent analyses showed that the surface-only group did not differ significantly in the overall proportions of distance traveled along arms and walls and in the open, but there were several significant interactions with trials attributable to the increase in the proportion of open travel and the accompanying decrease in arm travel during Trial Blocks 2 and 3 . The standard-arm controls showed no interactions with trial blocks, but they spent a greater proportion of their travel distance along maze arms than along walls or in the open $[F \mathrm{~S}(1,8)=12.9$ and 16.9 , respectively]. Comparing the two groups overall revealed that the rats in the surface-only group spent a smaller proportion of their locomotion in arm travel $[F(1,15)=$ 9.79] and a greater proportion in open travel $[F(1,15)=$ 20.1] than did the rats in the control group.

In short, the edge-only group and the surface-only group engaged in less arm travel and more open travel than did their respective controls, but the decrease in arm travel was significant only for the surface-only group $[F(1,15)=9.79]$. The proportion of wall travel did not differ for either group relative to their control groups. The control group results were quite similar, essentially replicating the results of Hoffman et al. (1999) in which arm travel dominated wall travel and travel in the open. The results also suggested the possibility that the standardarm rats in the slightly smaller arena $(3.2 \times 2.9 \mathrm{~m}$ vs. $3.6 \times 3.2 \mathrm{~m}$ ) traveled more along walls.

Effect of odor. The maze and floor of the arena were cleaned to remove possible odor cues before each of the sets of 4 or 2 rats was tested. To evaluate the possibility that ador trails of the rats tested early in a set might still have influenced the foraging behavior of the rats tested later in that set, we conducted ANOVAs on all dependent variables, comparing the early rats with the late rats. There were no significant differences in accuracy, speed, or travel pattern for any of the four groups.

\section{Discussion}

Because the efficiency and number of novel cup visits and the proportion of arm travel for the edge-only rats did not differ from its standard-arm controls, we conclude that the absence of the maze-arm substrate and one of 
a

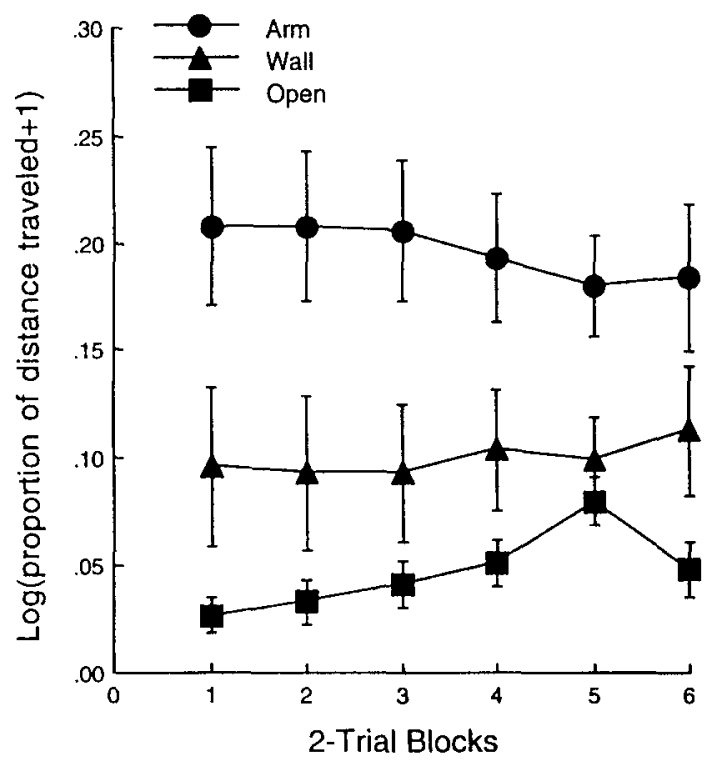

b

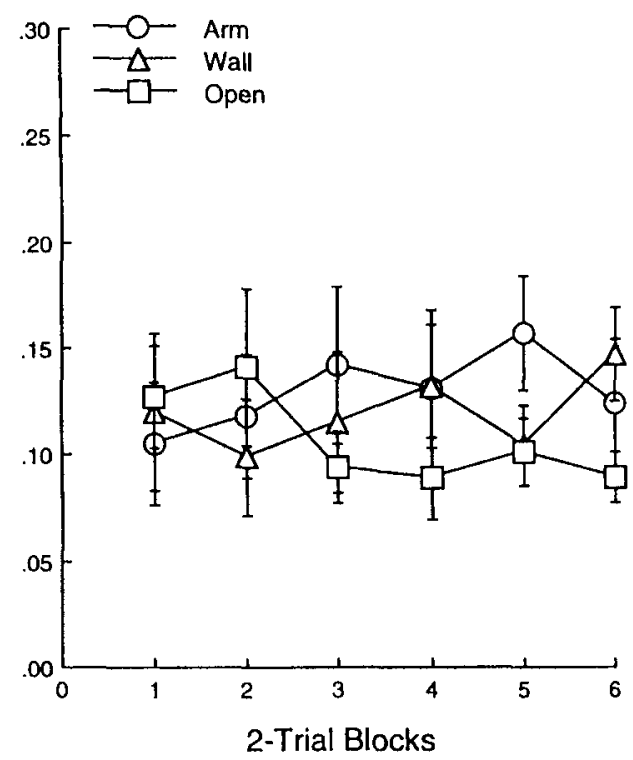

Figure 4. Mean proportion of distance traveled along maze arms and walls and in the open plotted by two-trial blocks for (a) the standard-arm group and (b) the edge-only group. Data are from Experiment 1.

the vertical edges had relatively minor effects on foraging behavior. In contrast, the removal of all vertical arm edges for the surface-only group resulted in markedly reduced arm travel, increased travel in the open, and poorer accuracy and less efficiency of foraging relative to the standard-arm control group. We conclude that the arm travel of rats on a floor maze is strongly influenced by some combination of visual and tactual cues from the vertical edges of the arm. Further support for the limited importance of substrate cues for arm travel is Hoffman et al.'s a

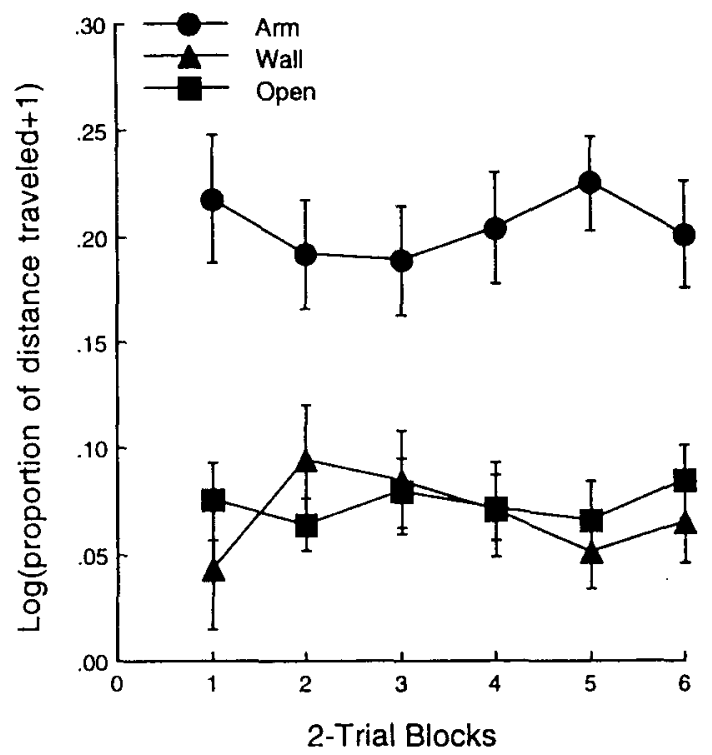

b

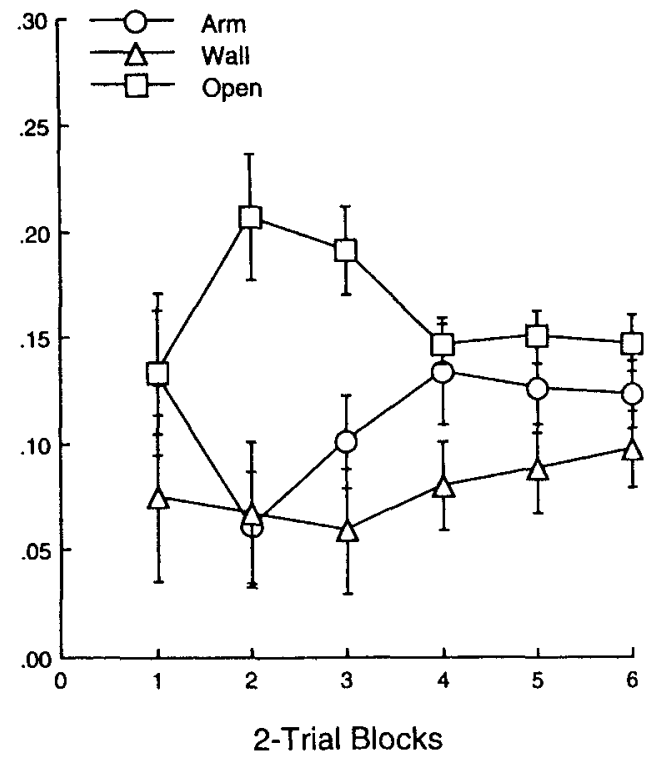

Figure 5. Mean proportion of distance traveled along maze arms and walls and in the open plotted by two-trial blocks for (a) the standard-arm group and (b) the surface-only group. Data are from Experiment 1 . 

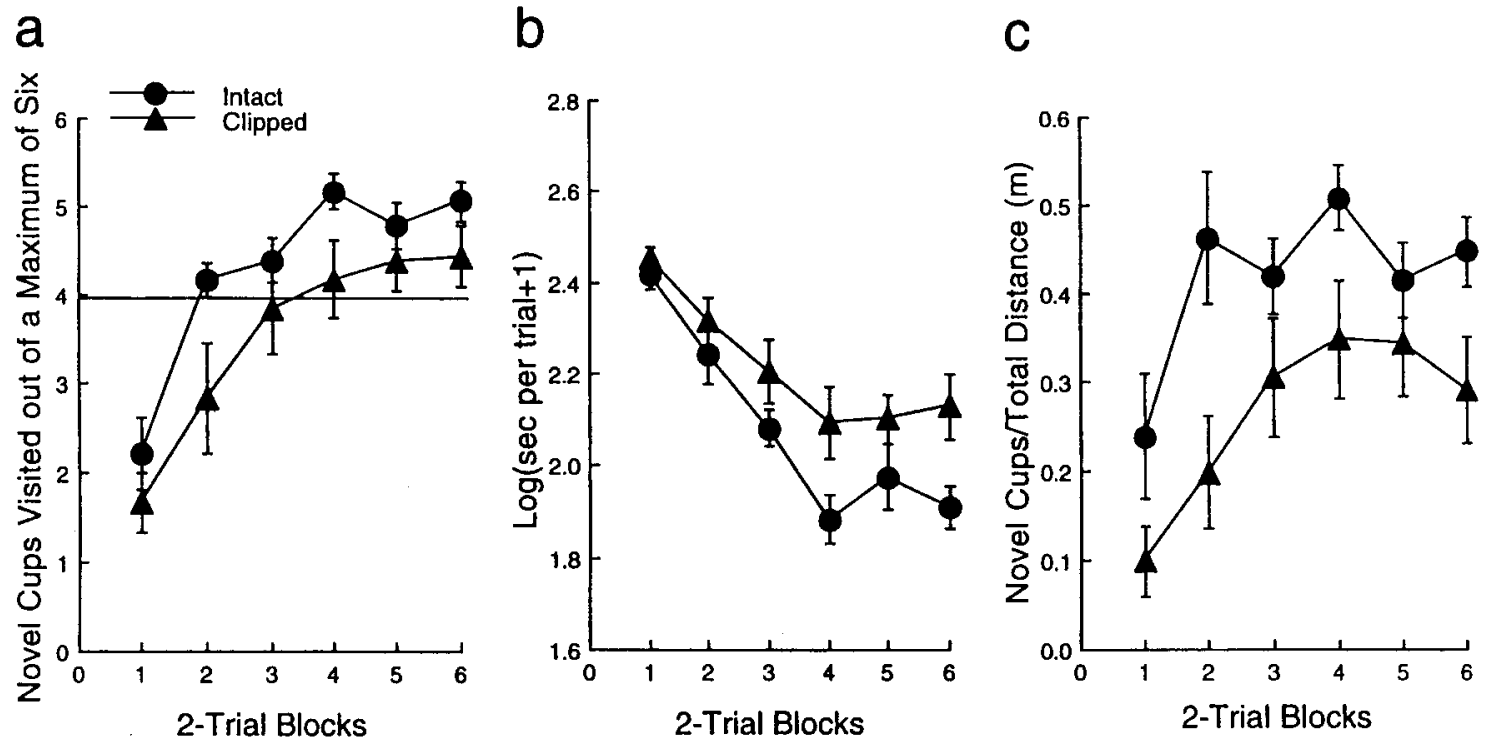

Figure 6. Accuracy, time, and efficiency measures of cup visits on a floor RAM by the rats with clipped and intact whiskers plotted by two-trial blocks: (a) mean number of novel cups visited in a maximum of six visits, (b) mean time to visit a maximum of six cups, and (c) mean novel cups visited per total distance traveled (cups/meter). The solid line in the first panel shows the number of novel cup visits expected by chance in six cup visits. The data are from Experiment 2.

(1999) report that more than $30 \%$ of arm travel in the case of the standard maze arm occurred alongside, rather than on top of, the arms. The arm surface alone increased distance traveled in the open.

\section{EXPERIMENT 2}

The results of Experiment 1 indicate that the vertical borders of the standard arms of our floor RAM were important in controlling arm travel, as well as the accuracy and efficiency of foraging. The question addressed in Experiment 2 was the extent to which tactual stimulation to the whiskers provided by the vertical edge is an important contributor to these effects. If it is, then interfering with tactual contact should interfere with arm travel and search efficiency. On the other hand, to the extent that visual input from the vertical borders dominated, we would expect little or no change due to tactual interference.

We interfered with tactual contact with the maze arms in two ways: (1) by trimming to approximately $0.5 \mathrm{~cm}$ the whiskers on one side of the head for a clipped group of rats and (2) by eliminating the vertical border from one side of each maze arm. Trimming the whiskers on only one side was intended to allow a test of our hypothesis while interfering less with daily activities, such as feeding, grooming, and moving about the environment. By removing only one of the arm borders, we attempted to clarify how any tactual deficit might work. If arm or wall travel has a contact-based component, we expected to see a directional bias toward moving in the direction in which the rat's intact whiskers could contact a vertical edge. A control group of intact rats was run on the same maze arms to take into account the effects of removing one border on search efficiency and locomotor patterns.

\section{Method}

\section{Subjects and Apparatus}

The subjects were 20 naive Sprague-Dawley rats, 90 days old at the beginning of the experiment, obtained, housed, and maintained in the same fashion as the rats in Experiment 1. The apparatus was the same as that for the standard-arm control group in Experiment 1 except that one border was removed from each maze arm (counterbalanced for side), and the arena was $3.6 \times 3.6 \mathrm{~m}$.

\section{Procedure}

The 20 rats were randomly divided into two groups. One subject from each group was eliminated for failing to find food on 2 of the first 3 days. For half of the rats in the clipped group, all whiskers on the right side of the head (projecting sideways, up, or down) were cut to a length of approximately $0.5 \mathrm{~cm}$. For the other half of the group, whiskers were cut on the left side. Each rat was wrapped in a towel, and surgical scissors were used to cut the whiskers; movement of the rat's head was restrained with the experimenter's free hand. The whiskers were maintained at approximately the same length during the experiment by trimming them approximately each 7 days. The remaining procedures were the same as those in Experiment 1 .

\section{Results}

The results are separated into data concerned primarily with (1) the effectiveness of the rats in finding all the food (number of novel cups visits out of a maximum of six visits, time to complete a trial, and efficiency of search) and (2) the patterns of locomotion shown in finding food 

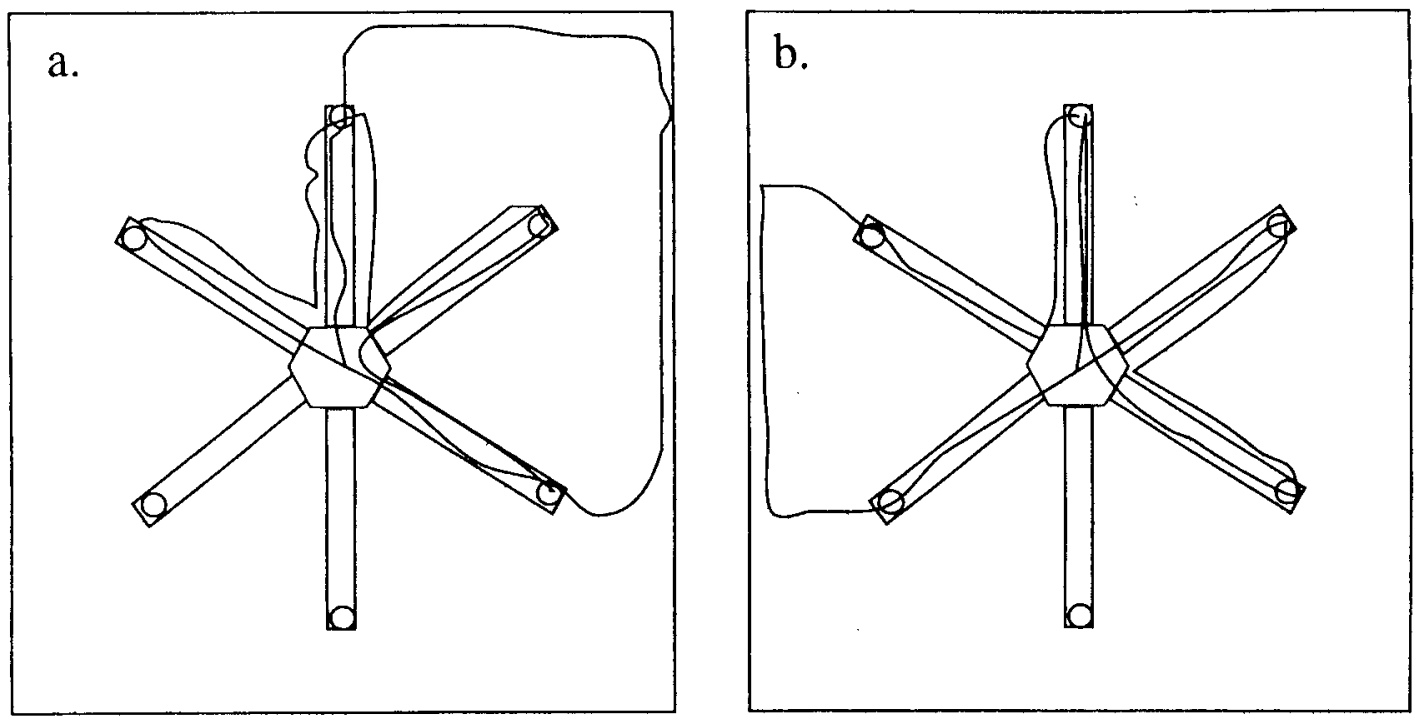

Figure 7. Modal routes on a floor RAM that represent the average measures over the last two blocks of trials in Experiment 2 for (a) clipped rats and (b) intact rats.

(proportion of adjacent cup visits, number of arcs, proportion of distance traveled along arms and walls and in the open, and the direction of travel).

\section{Cup Visits}

Novel cup visits. Figure 6 a shows that the number of novel cups visited in a maximum of six cup visits increased over days for both the clipped group and the intact group $[F(5,80)=31.7]$. Over all trial blocks, the rats in the intact group searched more novel cups than did the clipped rats $[F(1,16)=3.79]$. Although this difference did not reach significance, the direction of the difference was compatible with the fact that the intact group visited novel cups above our estimate of chance at asymptote during the last two blocks of trials $[t(8)=4.07]$, whereas the clipped group did not $[t(8)=1.34]$.

Time. Figure $6 \mathrm{~b}$ shows that both the clipped rats and the intact rats completed trials increasingly quickly over days $[F(5,80)=41.3]$. Over all trials, the intact rats were not significantly faster than the clipped rats in completing their trials $[F(1,16)=4.34, p=.054]$; however, they were significantly faster at asymptote (the last three blocks of trials of testing) $[F(1,16)=5.32]$.

Efficiency. Search efficiency was measured by dividing the number of novel cup visits by the total distance traveled (Figure $6 \mathrm{c}$ ). Efficiency increased markedly over trial blocks for both groups $[F(5,80)=10.4]$. Furthermore, the intact rats were more efficient (i.e., had a higher novel cups/distance ratio) than the clipped rats across all trial blocks $[F(1,16)=5.79]$.

\section{Locomotion Patterns}

Figure 7 shows a modal route taken by the rats in both groups (constructed from averages of the last 4 days, as outlined in Experiment 1). In each condition, the rats freely demonstrated their ability to travel on top of and alongside maze arms, in the open, and along the walls of the arena. The rats rarely traveled in the most energetically

Table 2

Mean Performance Measures and Standard Errors Averaged by Two-Trial Blocks for Experiment 2

\begin{tabular}{|c|c|c|c|c|c|c|c|c|c|c|c|c|c|}
\hline \multirow[b]{2}{*}{ Maze } & \multicolumn{2}{|c|}{ Block 1} & \multicolumn{2}{|c|}{ Block 2} & Block 3 & \multicolumn{2}{|c|}{ Block 4} & \multicolumn{2}{|c|}{ Block 5} & \multicolumn{2}{|c|}{ Block 6} & \multicolumn{2}{|c|}{ Overall } \\
\hline & $M$ & $S E$ & $M$ & $S E$ & $M S E$ & $M$ & $S E$ & $M$ & $S E$ & $M$ & $S E$ & $M$ & $S E$ \\
\hline \multicolumn{14}{|c|}{ ((a) Mean Proportion of Successive Visits to an Adjacent Cup } \\
\hline Clipp & .09 & .04 & .19 & .06 & $.29 \quad .05$ & .41 & .07 & .46 & .05 & .41 & .04 & .31 & .03 \\
\hline Intact Rats & .16 & .04 & .25 & .05 & $.20 \cdot .05$ & .34 & .06 & .34 & .08 & .40 & .05 & .28 & .03 \\
\hline \multicolumn{14}{|c|}{ (b) Mean Number of Arcs } \\
\hline Clip & 0.0 & 0.0 & 0.17 & 0.08 & 0.170 .08 & 0.44 & 0.18 & 0.39 & 0.11 & 0.33 & 0.18 & 0.25 & 0.0 \\
\hline Intact Rats & 0.17 & 0.08 & 0.17 & 0.12 & 0.060 .06 & 0.28 & 0.19 & 0.39 & 0.27 & 0.22 & 0.17 & 0.21 & 0.1 \\
\hline \multicolumn{14}{|c|}{ (c) Mean Distance Traveled Along Vertical Edge (in Meters) for Clipped Rats } \\
\hline Intac & 17.4 & 4.3 & .18 .7 & 4.6 & 13.32 .1 & 12.6 & 1.6 . & 1.1 .5 & 1.5 & 15.0 & 2.4 & 14.7 & 1.8 \\
\hline Clipped Whiskers & 15.2 & 2.0 & 10.7 & 3.1 & $13.0 \quad 1.9$ & 12.3 & 1.6 & 12.1 & 1.2 & 10.3 & 1.3 & 12.3 & 1.5 \\
\hline
\end{tabular}


a

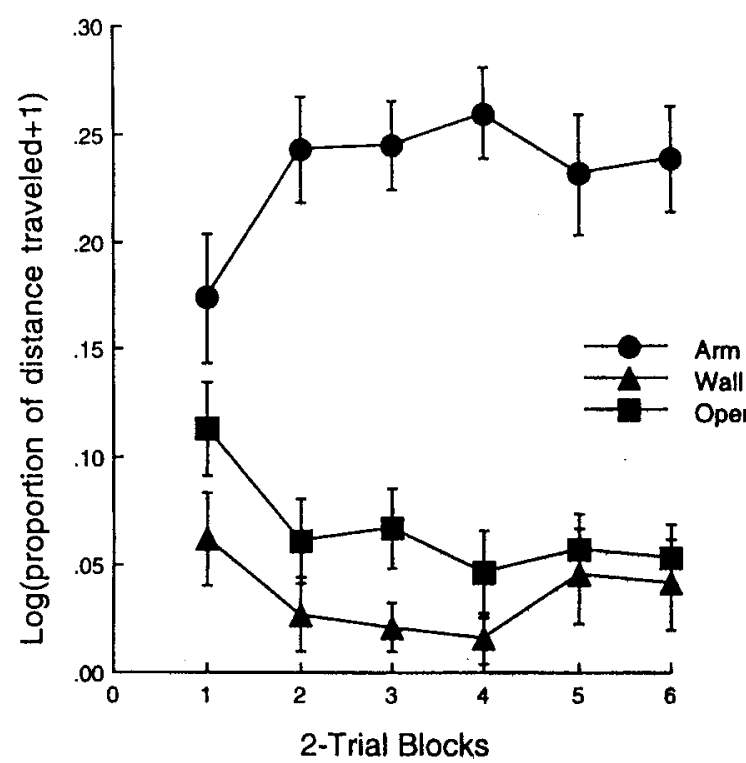

b

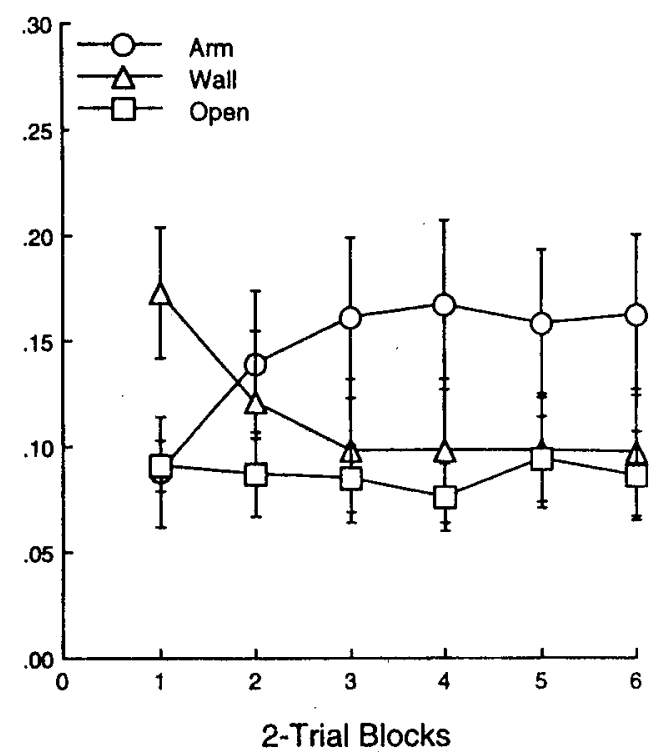

Figure 8. Mean proportion of distance traveled on a floor RAM by (a) intact rats and (b) clipped rats over two-trial blocks in Experiment 2.

efficient route in either condition. Instead, the modal route involved arm travel and, less frequently, travel along the arena wall.

Adjacent cup visits. Both the clipped rats and the intact rats significantly increased the proportion of successive visits to an adjacent cup over days (Table 2a) $[F(5,80)=10.7]$, suggesting that the rats tended to use an adjacent-arm response rule late in the experiment. The proportion of successive adjacent cup visits, though, did not differ significantly between the two groups $[F(1,16)<1]$.

Number of arcs. To test the possibility of distance minimizing explicitly, we recorded the number of arcs directly between adjacent food cups (see Table $2 b$ ). The mean total number of arcs was less than 1 for both groups, which did not differ from each other. The number of arcs did not change over trial blocks, and it differed significantly from the 5 arcs expected of an ideal distance minimizer $[t s(8)=50.2$ and 67.1 , for clipped and intact rats, respectively].

Proportion of distance traveled. An overall ANOVA showed no group difference but did show a difference among types of locomotion $[F(2,32)=11.3]$, changes over trial blocks $[F(5,80)=3.09]$, and two significant interactions (group $\times$ travel type, and trial block $\times$ travel type). Figure $8 \mathrm{a}$ shows that the proportion of travel along maze arms, along walls, and in the open differed for the intact rats $[F(2,16)=21.8]$. The intact rats traveled along arms significantly more than along walls and in the open $\left[F_{\mathrm{S}}(1,8)=29.9\right.$ and 17.5 , respectively $]$, and they traveled in the open significantly more than along the walls $[F(1,8)=13.5]$. Over trial blocks, the intact rats increased their proportion of travel along maze arms $[F(5,40)=$ $7.50]$ and decreased their proportion of travel in the open $[F(5,40)=6.81]$. Although the clipped rats in Figure 8b showed no significant difference in proportion of travel along arms, along walls, or in the open, there was a significant interaction between trial block and travel pattern $[F(10,80)=2.78]$. Over trial blocks, the clipped rats increased their proportion of arm travel and decreased wall travel $[F \mathrm{~s}(5,40)=3.90$ and 3.86 , respectively].

Comparing the clipped and intact groups on travel types, the intact rats traveled significantly more along maze arms than did the clipped rats but significantly less along the walls $[F s(1,16)=4.60$ and 6.01 , respectively]. There was no significant difference between the groups in proportion of travel in the open $[F(1,16)<1]$.

Direction of travel. Table $2 \mathrm{c}$ compares the average distance traveled along arms and walls in the direction in which the intact whiskers were closest to a vertical edge versus the direction in which the intact whiskers were farthest from the vertical edge. In terms of the maze arm, this direction was scored when (1) a rat was on top of the maze arm and the intact whiskers were closest to the intact border and (2) a rat was alongside the maze arm and the intact whiskers were closest to a side of the arm (both sides of the arm provided a minimum of 1.9-cm-high vertical stimulus, more than adequate for good whisker contact).

Although the data showed considerable similarity in direction traveled by the two groups during Trial Blocks $3-5$, an ANOVA overall six blocks of trials showed that the rats traveled significantly more often in the direction 
in which their intact whiskers could contact a vertical surface $[F(1,8)=8.32]$. There was no significant trial block effect or interaction.

Effect of odor. As in Experiment 1, we conducted ANOVAs on all dependent variables comparing the rats run early and those run late in a group of 4 . There were no significant differences in the performances of the early and late rats on accuracy, speed, efficiency, or travel pattern for either experimental group.

\section{Discussion}

Clipping the whiskers on one side of the head did not affect the rats daily behavior in informal observations, did not affect their average body weight, and did not decrease their total locomotion in the arena. However, in the arena, the clipped group was slower and less efficient in choosing novel cups than was the intact group. The number of novel cups chosen by the clipped rats in a maximum of six choices also never exceeded our (admittedly conservative) estimate of chance.

In terms of locomotor pattern, the clipped group traveled along arms significantly less and along walls significantly more than did the intact group. The clipped rats showed a pattern of locomotion similar to that of the noarm rats in Hoffman et al. (1999), although the clipped rats showed more open travel than did the no-arm rats. The presence of the arms may have encouraged the clipped rats to spend more time in the open.

Over all trial blocks, the clipped rats had a significant bias toward traveling along arms and walls in the direction that placed their intact whiskers next to the vertical surface. This effect, though, was not large enough to support a claim that whisker contact is the only determinant of wall or arm travel. It seems likely that visual cues play an important role as well, especially along arena walls and on the arena floor near the borders of maze arms (see also Roche \& Timberlake, 1998).

\section{EXPERIMENT 3}

The results of Experiments 1 and 2 suggest that the borders of standard maze arms and the arena wall share a common determinant of travel: a vertical extent the rats could tactually contact and, at least in the case of the arena wall and the border of the arm viewed from the arena floor, easily see. The purpose of Experiment 3 was to examine more directly the possibility of a similarity in function of arm and wall travel. It has been common to view the tendency to remain near and travel along walls in an open arena (thigmotaxis) as due to the effects of fear in keeping the rat close to the "cover" of the wall (e.g., Barnett, 1975). The effect of novelty, illumination intensity, and age in increasing the amount of wall travel has been seen as support for this interpretation (Valle, 1970, 1971).

An alternative hypothesis is that arm travel and wall travel are both related to trail following and thus are likely to share underlying sensorimotor mechanisms and similar motivations. This would follow from the hypothesis that, on an evolutionary time scale, trail following for a social animal, such as the rat, was selected as a general tactic that served many functions, including increased safety, access to social information about food locations, and location of estrus females by interested males ( $\mathrm{Cal}$ houn, 1962). With regard to safety, consider that natural trails tend to follow vertical cover, such as tree trunks, fences, and bushes (Calhoun, 1962), that can interfere with attacks by aerial predators, whereas the unimpeded continuous nature of trails provides easy escape routes from large ground predators. This view suggests that maze arms should also be effective in guiding travel in fear-inducing situations. Although they lack the visual extent of walls, maze arms do have tactual borders on both sides and unimpeded extent.

In Experiment 3, we evaluated the relative merits of the wall-hugging (fear) hypothesis of arena locomotion versus the general trail-following hypothesis outlined above in accounting for behavior during the first 4 days of exposure to a floor RAM arena. Four groups were formed by the factorial combination of the presence and the absence of standard floor RAM maze arms and the conditions of bright and dim illumination. The fear hypothesis predicts an effect of bright light in reducing locomotion and food finding while increasing wall travel, relative to arm and open travel. As fear decreases over trials, food finding should increase along with increased travel in the open.

In contrast, the trail-following hypothesis predicts that the presence of arms should increase arm and open travel as well as effective food finding and that bright light should interfere relatively little with arm travel. The rats were tested for 4 days in order to enable us to focus on the initial reactions of the rats to the stimulus conditions, a time when fear would be expected to predominate and it would be difficult to argue that the results were due primarily to reinforcement of specific locomotor patterns by finding food.

\section{Method}

\section{Subjects and Apparatus}

The subjects were 40 naive Sprague-Dawley rats, 90 days old at the beginning of the experiment. The rats were randomly divided into four groups of 10 . The four groups represented the factorial combination of two levels of illumination (bright, 300-W overhead bulb, and $\operatorname{dim}, 25-\mathrm{W}$ overhead bulb; both bulbs were mounted $7 \mathrm{ft}$ above the center of the arena) with the presence and absence of maze arms. All conditions used the center platform and food cups placed in the same spatial configuration as those in Experiment 1 . The arena size was $3.6 \times 3.6 \mathrm{~m}$.

\section{Procedure}

The rats were run for four trials using the same procedures as those in Experiment 1. One subject from the bright-light/no-arms group was eliminated for failing to leave the center platform on 2 of the first 3 days. Most of the measures were the same as those in 
a

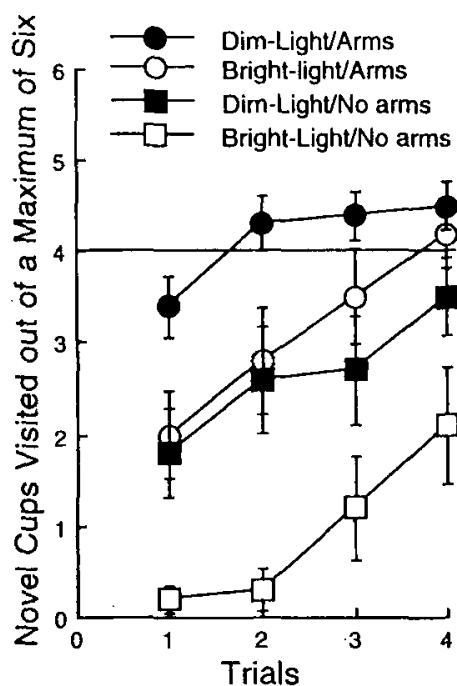

b

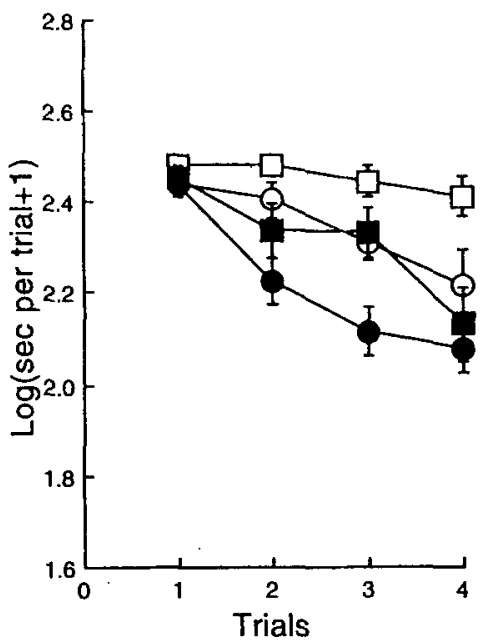

C

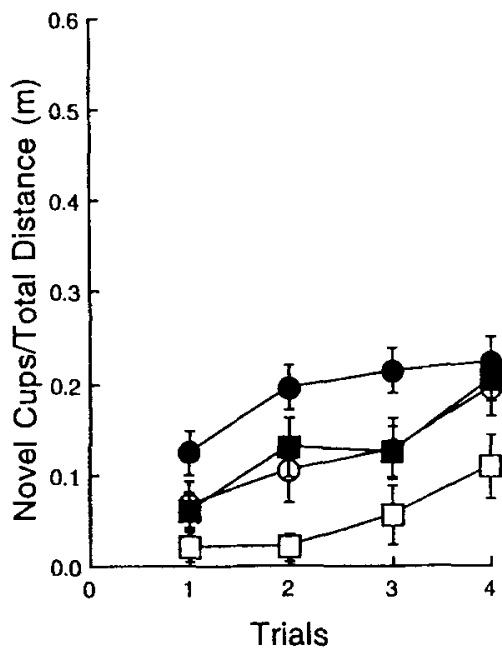

Figure 9. Accuracy, time, and efficiency measures of cup visits plotted by trials for Groups dim-light/arms, brightlight/arms, dim-light/no-arms, and bright-light/no-arms in Experiment 3: (a) mean number of novel cups visited in a maximum of six visits, (b) mean time to visit a maximum of six cups, and (c) mean novel cups visited per total distance traveled (cups/meter). The solid line in panel a shows the number of novel cup visits expected by chance in six cup visits.

previous experiments. Because proportions of distance traveled are often highly variable during initial trials due to small total amounts of travel, we reported absolute, rather than proportional, distances of wall travel and nonwall travel (the latter included travel in the open and, in the case of the arm conditions, travel along the arms).

\section{Results}

The results are separated into data concerned primarily with (1) the effectiveness of the rats in finding all the food (number of novel cup visits out of a maximum of six visits, time to complete a trial, and efficiency of search) and (2) the patterns of locomotion shown in finding food (proportion of successive adjacent cup visits, number of arcs, and absolute distance traveled along arms and walls and in the open).

\section{Cup Visits}

Novel cup visits. As shown in Figure 9a, the number of novel cup choices in a maximum of six visits increased significantly over the first 4 days $[F(3,105)=$ 16.3]. Overall, the dim-light rats visited significantly more novel cups than did the bright-light rats $[F(1,35)=$ 17.1], and the rats in the arms groups visited significantly more novel cups than did the rats in the no-arms groups $[F(1,35)=31.3]$. There were no significant interactions; however, when maze arms were present, the difference between illumination conditions disappeared by Trial 4 $[F(1,18)<1]$.

Time. Figure $9 \mathrm{~b}$ shows that the $\log$ time to visit six cups decreased over days $[F(3,105)=25.4]$. Overall, the dim-light rats were faster at visiting six cups than the bright-light rats $[F(1,35)=17.6]$, and the rats in the arms groups were faster than the rats in the no-arms groups $[F(1,35)=10.8]$. There also was an interaction between illumination group and days $[F(3,105)=4.4]$ produced by the absence of a difference among the groups on the Ist day.

Efficiency. Efficiency was measured as in Experiment 1 (novel cups/total distance). On the basis of this measure, Figure $9 \mathrm{c}$ shows that (1) the rats became more efficient over days $[F(3,105)=19.8],(2)$ the rats in the dim-light groups were significantly more efficient than the rats in the bright-light groups $[F(1,35)=12.3]$, and (3) the rats in the arms groups were significantly more efficient than the rats in the no-arms groups $[F(1,35)=$ 10.2]. Except for the lagging bright-light/no-arms group, the differences in efficiency due to illumination and presence of maze arms disappeared by Day 4 (see Roche \& Timberlake, 1998, for similar disappearance of initial differences in efficiency in floor RAMs with different arm configurations).

\section{Locomotion Pattern}

Figure 10 shows a modal route taken by the rats in each group (based on the last two blocks of trials as outlined in Experiment 1). In each condition, the rats traveled on top of and alongside maze arms, in the open, and along the walls of the arena. The rats did not travel in the most energetically efficient route in any condition. Instead, they tended to travel along maze arms when they were available and along the arena wall when arms were not present. 

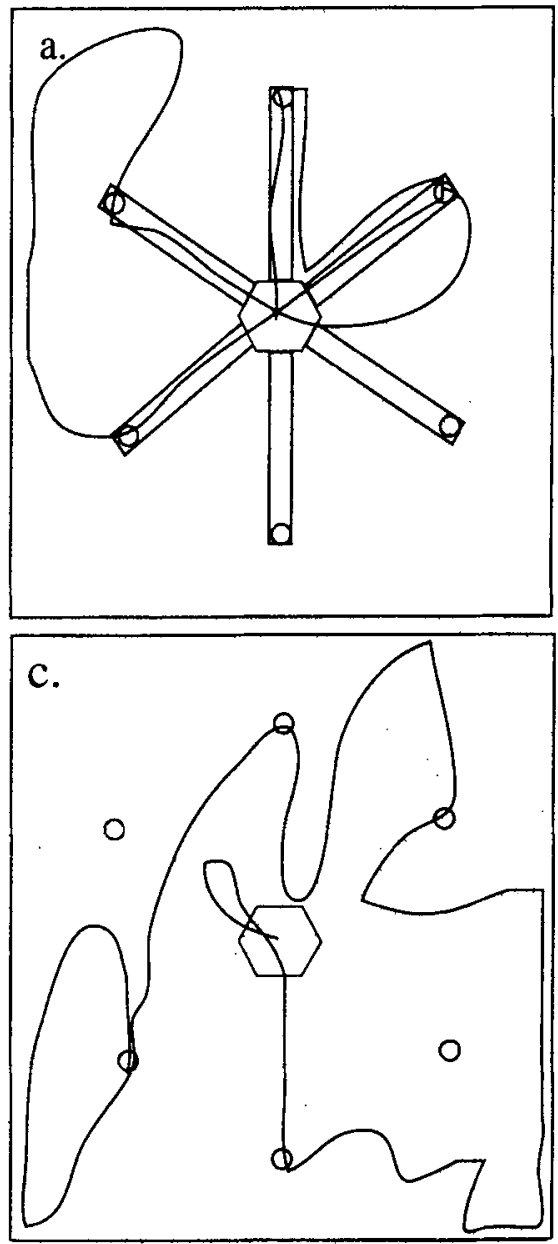
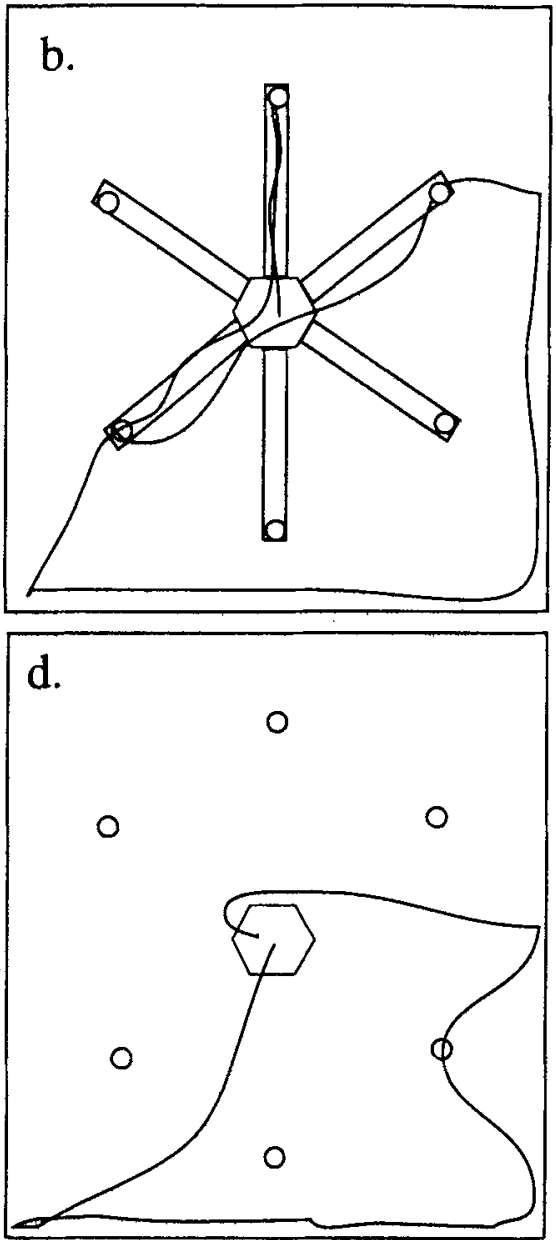

Figure 10. Modal routes with food cups arranged in a floor RAM configuration over the last two trials in Experiment 3 for (a) the dim-light/arms group, (b) the bright-light/arms group, (c) the dimlight/no-arms group, and (d) the bright-light/no-arms group.

Adjacent cup visits. The rats increased the proportion of adjacent cup visits over days $[F(3,105)=4.94]$. The rats in the dim-light conditions visited proportionally more adjacent cups than did the rats in the bright-light conditions $[F(1,35)=12.1]$ (Table 3a). A similar, though not significant, difference occurred between arms and noarms groups $[F(1,35)=4.07]$.

Number of arcs. Table $3 \mathrm{~b}$ shows the mean number of arcs for each group was less than 0.3 , well under the 5 arcs of an ideal minimizer. The rats in dim-light conditions did, though, travel significantly more arcs than the rats in the bright-light conditions $[F(1,35)=4.93]$. There was no effect of arms on number of arcs $[F(1,35)=2.48]$.

Absolute distance traveled. The absolute distances traveled along the wall and not along the wall are shown in Figures $11 \mathrm{a}$ and $11 \mathrm{~b}$, respectively (absolute distance traveled was used because some rats moved very little in the initial trials, leading to very high variance in the proportion of distance-traveled measure). Overall, bright light obviously inhibited nonwall travel $[F(1,37)=7.44]$, whereas the presence of arms increased both wall travel and nonwall travel $\left[F_{\mathrm{S}}(1,37)=5.93\right.$ and 15.5 , respectively]. There was a significant interaction between illumination and trials for nonwall travel $[F(3,111)=2.83]$. For nonwall travel in the no-arms condition, bright illumination had a marked inhibitory effect. In contrast, in the arms condition, bright light inhibited nonwall travel only on the 1st day. Wall travel also showed an initial inhibitory effect of bright illumination; however, when arms were present, brightness produced a marked facilitatory effect. The result was a significant interaction between arm presence and brightness $[F(3,111)=5.01]$. In $\mathrm{dim}$ light, the presence of maze arms had no significant effect on wall travel.

Effect of odor. There were no significant differences between the rats run early and those run late in a trial on any of the measures for the arms and no-arms groups. Furthermore, there were no significant differences between early and late rats in bright-light or dim-light conditions on the measures of novel cups and time. However, 
Table 3

Mean Performance Measures and Standard Errors by Trial for Experiment 3

\begin{tabular}{|c|c|c|c|c|c|c|c|c|c|c|}
\hline \multirow[b]{2}{*}{ Condition } & \multicolumn{2}{|c|}{ Day 1} & \multicolumn{2}{|c|}{ Day 2} & \multicolumn{2}{|c|}{ Day 3} & \multicolumn{2}{|c|}{ Day 4} & \multicolumn{2}{|c|}{ Overall } \\
\hline & $M$ & $S E$ & $M$ & $S E$ & $M$ & $S E$ & $M$ & $S E$ & $M$ & $S E$ \\
\hline \multicolumn{11}{|c|}{ (a) Mean Proportion of Successive Visits to an Adjacent Cup } \\
\hline Dim light/Arms & .27 & .07 & .28 & .07 & .32 & .05 & .38 & .07 & .31 & .04 \\
\hline Bright light/Arms & .10 & .06 & .17 & .08 & .22 & .06 & .31 & .07 & 20 & .05 \\
\hline Dim light/No arms & .24 & .08 & .23 & .06 & .23 & .06 & .33 & .08 & .26 & .04 \\
\hline Bright light/No arms & .0 & .0 & .04 & .04 & .15 & .08 & .15 & .06 & .09 & .04 \\
\hline \multicolumn{11}{|c|}{ (b) Mean Number of Arcs } \\
\hline Dim light/Arms & 0.10 & 0.10 & 0.30 & 0.15 & 0.70 & 0.26 & 0.30 & 0.15 & 0.35 & 0.11 \\
\hline Bright light/Arms & 0.0 & 0.0 & 0.20 & 0.20 & 0.0 & 0.0 & 0.20 & 0.13 & 0.10 & 0.08 \\
\hline Dim light/No arms & 0.0 & 0.0 & 0.20 & 0.13 & 0.20 & 0.13 & 0.20 & 0.13 & 0.15 & 0.06 \\
\hline Bright light/No arms & 0.0 & 0.0 & 0.0 & 0.0 & 0.0 & 0.0 & 0.22 & 0.15 & 0.06 & 0.04 \\
\hline
\end{tabular}

there were significant differences in efficiency between early and late rats for both the bright-light and dim-light groups $\left[F_{\mathrm{S}}(1,8)=13.8\right.$ and 6.73 , respectively]. Interestingly, in the bright-light condition, the early rats were more efficient than the late rats, whereas in the dim-light condition, the late rats were more efficient than the early rats. Also, in the bright-light groups, there was a significant difference in distance traveled along the wall $[F(1,8)=$ 8.51 ], with the early rats traveling less around the wall than the late rats. It is worth noting that these results are for 4 days only. Had the present rats received more trials, these differences probably would have disappeared.

\section{Discussion}

The data better supported the trail-following hypothesis of floor RAM locomotion than the simple-fear hy- pothesis. The presence of maze arms reduced the inhibitory effects of bright illumination on both wall travel and nonwall travel. However, bright illumination initially reduced accuracy, time, and efficiency of cup visits, relative to dim illumination, even when there were maze arms, though these effects disappeared by Day 4 . The presence of maze arms facilitated locomotion and efficient food finding in both bright-light and dim-light environments. The present data, combined with those for the no-arms rats in Hoffman et al. (1999), indicate that both arm travel and wall travel occur in the presence of fear and novelty and that both arm travel and wall travel play roles in finding food. These results would be expected if both were examples of trail following and if the mechanisms supporting trail following had been selected for over evolutionary time to serve multiple functions.

b

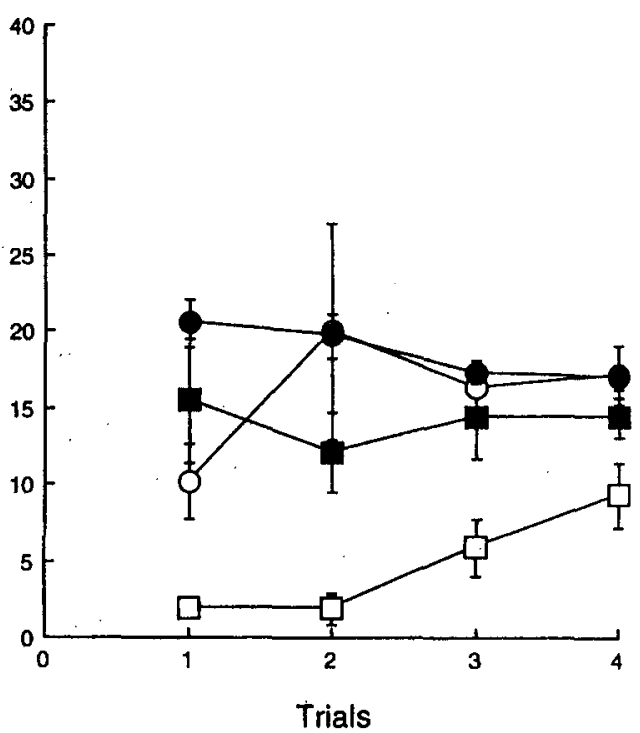

Figure 11. Mean absolute distance traveled plotted by trials and by travel type for (a) travel along walls and (b) travel away from the walls. Data are from Experiment 3. 


\section{GENERAL DISCUSSION}

Several conclusions follow from these floor RAM experiments. First, the presence of a vertical surface connected to the substrate is an important determinant of arm and wall travel in an arena (see also Peden \& Timberlake, 1990; Roche \& Timberlake, 1998). Arm travel was not facilitated by the simple presence of a horizontal surface differing in reflectance and texture from the remainder of the arena floor. Although rats clearly are able to discriminate among maze arms on the basis of such surface cues (Finger \& Frommer, 1968; Reyes, Finger, \& Frye, 1973), only the presence of a vertical edge appears to produce extensive arm travel and efficient search on a floor RAM.

Second, a primary channel for sensing a low vertical edge appears to be tactual contact with the rats' whiskers. When whiskers were clipped short on one side of the head, the rats showed significantly less total arm travel, greater wall travel, and less efficient foraging than did a control group with the same maze configuration. Clipped rats also showed a bias toward travel with their intact whiskers closest to vertical surfaces. Though orientation based on whisker contact was clearly not the only important determinant of locomotion, these data suggest that its contribution may have been underestimated in current conceptions of maze behavior.

These data in no way deny that rats use other than tactual cues, such as visual or olfactory stimuli, in foraging for food. For example, Roche and Timberlake (1998), working with the same configuration of central platform and food cups as was used in the present experiments, but without maze arms, showed a marked effect of 46-cmhigh striped landmarks in guiding distance-minimizing search in the form of arcs between the food cups. However, when maze arms and visual landmarks were both available, the rats arm traveled rather than following direct arcs between the food cups.

Data from traditional maze studies also support a role for continuity and length of tactual and visual stimulation in maze locomotion. For example, alley length is important in speed of acquisition (Timberlake, 1983), elimination of blind alleys (White \& Tolman, 1923), and development of working memory for radial arms (Brown \& Huggins, 1993). Roche and Timberlake (1998) also showed that when the shortened arms of a floor RAM extended only halfway to food, both travel along the arms and initial efficiency of foraging decreased. Finally, the present data indicate a role for olfactory cues in controlling initial locomotion in the absence of arms and in $\mathrm{dim}$ illumination.

The third conclusion supported by the present data is that the presence of arms facilitates the emergence of movement away from the wall as well as successful and efficient food finding. This facilitatory effect was most noticeable in a novel environment and in the presence of traditional fear-inducing stimuli, such as bright lighting.
Over trials, though, the initial advantage of having maze arms aligned with food cups diminished. In the dim-light condition of Experiment 3, the advantage of having arms present disappeared by Trial 4 . Similarly, Roche and Timberlake (1998) showed that the advantage of rats with maze arms leading directly to food cups, rather than between food cups, partway to food cups, or in a zig-zag to food cups, disappeared by Trial Block 4 .

Finally, the present data argue that, on the basis of definition rather than its typical usage, thigmotaxis may indeed contribute to both arm travel and wall travel in an arena. Thigmotaxis literally means an orientation movement based on contact, rather than referring to a fearful hiding against the wall that is often implied. It appears that arm travel can be markedly affected by whisker contact (see also Munn, 1950, pp. 42-43). However, that rats with half their whiskers clipped decreased arm travel but increased wall travel suggests that wall travel is also controlled considerably by visual input and, perhaps, body contact.

Taken together with previous floor maze data (Hoffman et al., 1999; Roche \& Timberlake, 1998), the present results support the hypothesis that locomotion on a floor RAM and, by extension, the standard elevated RAM is influenced by the sensory mechanisms of trail following more than by the tactics of distance minimizing, central-place search, or random search. In standard elevated or enclosed laboratory mazes, the influence of trail following cannot be distinguished from that of other tactics. However, that rats on a floor RAM persisted in arm travel despite its inefficiency is evidence against the singular importance of optimality rules and is evidence for the initial importance of relatively automatic control of locomotion by the tactual mechanisms supporting arm travel.

The tactic and mechanisms of efficient trail following may contribute to several common maze phenomena by providing a basis of unlearned arm and alley travel (Timberlake, 1983). The tendency to exhaustively follow vertical stimuli, such as maze walls, as shown in the unbaited-arms condition of Experiment 2 in Hoffman et al. (1999), could help account for spontaneous alternation in simple choice mazes (Dember \& Fowler, 1958; Douglas, 1966), the tendency of rats in a Dashiell (1930) maze to travel a variety of different routes from start to goal, and the "patrolling" behavior of rats shown in an unbaited enclosed alley maze (Bättig, Driscoll, Schlatter, \& Uster, 1976; Uster, Bättig, \& Nägeli, 1976) and on an unbaited elevated RAM (Timberlake \& White, 1990).

Finally, our results suggest the possibility that the common practice of putting low borders on elevated RAMs for rats may have arisen because they allow mechanisms of trail following to better contribute to rapid locomotion. We have heard researchers claim that low borders prevent rats from hanging their heads over the edge of the arm, thereby threatening to jump and in general taking too long to complete the maze. Viewed in the light of the present evidence, rats may hang their heads over the edge of the arm to make better whisker contact with the verti- 
cal side of the maze arm. Adding a border makes this contact more readily available, so hanging the head is less necessary. If our interpretation is correct, this would predict faster acquisition and performance in elevated mazes with borders than in elevated mazes without borders.

In sum, the data on floor RAM behavior support the hypothesis that rats have a dominant locomotor tactic of traveling along trails based partly on whisker contact with continuous low vertical surfaces and partly on visual perception of taller vertical stimuli. This seems an ideal set of mechanisms for a partially nocturnal, trail-making, group-foraging species that lives socially both above and below ground and uses trails to enhance safety and promote the location of food and conspecifics. Even in the absence of already-established trails, rats would be expected to traverse terrain by following low vertical edges and a string of tall vertical landmarks. Such an ecological analysis suggests that other species may differ in some dominant tactics and mechanisms of locomotion. Thus, although a variety of species apparently can forage efficiently on an elevated RAM, they may differ widely in their amount of arm travel given access to a floor RAM.

\section{REFERENCES}

Barnett, S. A. (1975). The rat. Chicago: Chicago University Press. Bättig, K., Driscoll, P., Schlatter, J., \& Uster, H. J. (1976). Effects of nicotine on the exploratory locomotion patterns of female Roman high- and low-avoidance rats. Pharmacology, Biochemistry \& Behavior, 4, 435-439.

Brown, M. F., \& Huggins, C. K. (1993). Maze-arm length affects a choice criterion in the radial-arm maze. Animal Learning \& Behavior, 21, 68-72.

CALHOUN, J. B. (1962). The ecology and sociology of the Norway rat (Publication No. 1008). Bethesda, MD: U.S. Department of Health, Education and Welfare.

Dashiell, J. F. (1930). Direction orientation in maze running by the white rat. Comparative Psychology Monographs, 7 (No. 272).

DEMBER, W. N., \& FoWLER, H. (1958). Spontaneous alternation behavior. Psychological Bulletin, 55, 412-428.

Douglas, R. J. (1966). Cues for spontaneous alternation. Journal of Comparative \& Physiological Psychology, 62, 171-183.
Finger, S., \& Frommer, G. P. (1968). Effects of somatosensory thala$\mathrm{mic}$ and cortical lesions on roughness discrimination in the albino rat. Physiology \& Behavior, 3, 83-89.

GALEF, B. G., JR., \& BUCKLEY, L. L. (1996). Use of foraging trails by Norway rats. Animal Behaviour, 51, 765-771.

Hoffman. C. M., Timberlake, W., Leffel, J., \& Gont, R. (1999). How is radial arm maze behavior in rats related to locomotor search tactics? Animal Learning \& Behavior, 27, 426-444.

MunN, N. L. (1950). Handbook of psychological research on the rat. New York: Houghton Mifflin.

Peden, B. F., \& Timberlake, W. (1990). Environmental influences on flank and urine marking by male and female rats (Rattus norvegicus). Journal of Comparative Psychologv, 104, 122-130.

Reyes, R., Finger, S., \& Frye, J. (1973). Serial thalamic lesions and tactile discrimination in the rat. Behavioral Biology, 8, 807-813.

Roche, J. P., \& Timberlake, W. (1998). The influence of artificial paths and landmarks on the foraging behavior of Norway rats (Rattus norvegicus). Animal Learning \& Behavior, 26, 76-84.

Simon, P., Dupuis, R.. \& Costentin, J. (1994). Thigmotaxis as an index of anxiety in mice: Influence of dopaminergic transmissions. Behavioural \& Brain Research, 61, 59-64.

Tel.le, H. J. (1966). Beitrag zur Kenntnis der Verhaltensweise von Ratten, vergleichend dargestellt bei, Rattus norvegicus und Rattus rattus [Contribution to knowledge of the behavior of rats, a comparative study of Rattus norvegicus and Rattus rattus]. Zeitschrift fur Angewandte Zoologie, 48, 97-129.

TimBERLAKE, W. (1983). Appetitive structure and straight alley running. In R. L. Mellgren (Ed.), Animal cognition and behavior (pp. 165221). New York: North-Holland.

Timberlake, W., \& White, W. (1990). Winning isn't everything: Rats need only food deprivation not food reward to traverse a radial arm maze efficiently. Learning \& Motivation, 21, 153-163.

Uster, H. J., BäTTIG, K., \& NÄGELI, H. H. (1976). Effects of maze geometry and experience on exploratory behavior in the rat. Animal Learning \& Behavior, 4, 84-88.

VALLE, F. P. (1970). Effects of strain, sex, and illumination on open field behavior of rats. American Journal of Psychology, 83, 103-111

VALLE, F. P. (1971). Rats' performance on repeated tests in the open field as a function of age. Psychonomic Science, 23, 333-335.

White, A. E., \& Tolman, E. C. (1923). A note on the elimination of short and long blind alleys. Journal of Comparative Psychologv, 3 , 327-331.

(Manuscript received August 19, 1998; revision accepted for publication June 14, 1999.) 